\title{
Preliminary results of an analytical model to determine the internal quantum efficiency of a predictable quantum efficient detector
}

\author{
Resultados preliminares de un modelo analítico para la \\ determinación de la eficiencia cuántica interna de un detector \\ cuántico, eficiente y predecible
}

\author{
E. Borreguero ${ }^{1 *}$, A. Ferrero ${ }^{1, S}$, C. K. Tang 2 , J. Gran ${ }^{2}$, A. Pons ${ }^{1, S}$, J. Campos ${ }^{1, S}$ and M. L. \\ Hernanz ${ }^{1, \mathrm{~S}}$. \\ 1. Instituto de Óptica "Daza de Valdés" (IO, CSIC), Serrano 121, 28006 Madrid, Spain \\ 2. Justervesenet (JV), PO Box 170, 2027 Kjeller, Norway \\ (*) E-mail: elisa.borreguero@csic.es \\ S: miembro de SEDOPTICA / SEDOPTICA member
}

\author{
Received: 01/12/2016 Accepted:21/11/2017
}

DOI: $10.7149 /$ OPA.50.4.49027

\begin{abstract}
:
The potential of predictable quantum efficient detectors (PQEDs) as optical radiant power primary standard, based on photoelectric effect in silicon semiconductor, has been proved. Until now, the internal quantum efficiency (IQE) of a PQED is only predicted, from the design and setup parameters of the two photodiodes of this radiometer, by means of simulation software for semiconductor devices. This work presents, as alternative method, an analytical model based on Ferrero et al. photocurrent analysis, which considers the different internal regions of the photodiode and the characteristics of the incident beam. The IQE grows with the reverse bias voltage applied to the photodiodes and the lifetime of the charge carriers in the bulk, while IQE decreases when the surface recombination velocity and the doping concentration of the substrate are increased. The IQE results of the analytical model are similar to simulations for wavelengths between $400 \mathrm{~nm}$ and $700 \mathrm{~nm}$. Moreover, the analytical model predicts an increase of the IQE with the irradiance, at certain levels of optical power due to the supra-responsivity of the photodiode.
\end{abstract}

Key words: internal quantum efficiency, photodiode, PQED.

\section{RESUMEN:}

Los detectores de eficiencia cuántica predecible (PQED) han demostrado su potencial para llegar a desarrollar un patrón primario de flujo radiante, basado en el efecto fotoeléctrico del silicio como material semiconductor. Hasta ahora, la eficiencia cuántica interna (IQE) de un radiómetro PQED se determina, a partir de parámetros de diseño y montaje de los dos fotodiodos que lo constituyen, mediante programas de simulación de dispositivos semiconductores. Este trabajo presenta como alternativa un modelo analítico, basado en el estudio de la fotocorriente de Ferrero et al., que considera las distintas regiones internas del fotodiodo y las características del haz incidente. La IQE aumenta con el voltaje de polarización inversa aplicado al PQED y el tiempo de vida de los portadores de carga, mientras que disminuye al aumentar la velocidad de recombinación de superficie y la concentración de impurezas dopantes en el sustrato. Los resultados de IQE obtenidos con el modelo y las simulaciones son similares para longitudes de onda entre $400 \mathrm{~nm}$ y $700 \mathrm{~nm}$. Además, el modelo analítico predice un aumento de la IQE con la irradiancia a partir de ciertos niveles, consecuencia de la aparición de suprarresponsividad en los fotodiodos.

Palabras clave: eficiencia cuántica interna, fotodiodo, PQED. 


\section{REFERENCES AND LINKS / REFERENCIAS Y ENLACES}

[1] EMRP European project, "Candela: Towards quantum based photon standards," http://www.quantumcandela.org

[2] EMRP European project, "New primary standard and traceability for radiometry," https://www.inrim.eu/research-development/bulk-nano/photonics/newstar/project

[3] T. E. Hansen, "Silicon UV-photodiodes using natural inversion layers", Physica Scripta 18, 471-475 (1978).

[4] M. Sildoja, F. Manoocheri, M. Merimaa, E. Ikonen, I. Müller, L. Werner, J. Gran, T. Kübarsepp, M. Smîd, M.L. Rastello, "Predictable quantum efficient detector: I. Photodiodes and predicted responsivity", Metrologia 50, 385-394 (2013).

[5] I. Müller, U. Johannsen, U. Linke, L. Socaciu-Siebert, M. Smîd, G. Porrovecchio, M. Sildoja, F. Manoocheri, E. Ikonen, J. Gran, T. Kübarsepp, G. Brida, L. Werner, "Predictable quantum efficient detector: II. Characterization and confirmed responsivity", Metrologia 50, 395-401 (2013).

[6] J. Gran, T. Kübarsepp, M. Sildoja, F. Manoocheri, E. Ikonen and I. Müller, "Simulations of a predictable quantum efficient detector with PC1D", Metrologia 49, 130-134 (2012).

[7] A. Ferrero, J. Campos, A. Pons, A. Corrons, "New model for the internal quantum efficiency of photodiodes based on photocurrent analysis", Applied Optics 44, 208-216 (2005).

[8] M. Sildoja, F. Manoocheri and E. Ikonen, "Reflectance calculations for predictable quantum efficient detector", Metrologia 46, 151-154 (2009).

[9] A. S. Grove, Physics and technology of semiconductors devices. Willey, New York (1967).

[10] M. A. Green, "Self-consistent optical parameters of intrinsic silicon at $300 \mathrm{~K}$ including temperature coefficients", Solar Energy Materials \& Solar Cells 92, 1305-1310 (2008).

[11] C. M. Herzinger, B. Johs, W. A. McGaham, J. A. Woollam and W. Paulson "Ellipsometric determination of optical constants for silicon and thermally grown silicon dioxide via a multi-sample, multiwavelength, multi-angle investigation", Journal of Applied Physics 83, 3323-3336 (1998).

\section{Introduction}

In the last years, within the European joint projects "Candela: Towards quantum based photon standards" (qu-Candela) [1] and "New primary standards and traceability for radiometry" (NEWSTAR) [2], with the development and improvement of self-induced junction photodiodes [3] and the packaging in a wedged trap configuration, a high efficient light-detecting instrument called predictable quantum efficient detector (PQED) $[4,5]$ has been achieved. The potential of PQEDs as optical radiant power primary standard, based on photoelectric effect in silicon semiconductor, has been proved through comparison to the present primary standard, the cryogenic electrical substitution radiometer.

A primary standard bonds a quantity to fundamental constants or SI units, without being compared to other standards. A PQED links the optical radiant power to fundamental constants through its internal quantum efficiency (IQE). IQE represents the number of electrons that contribute to the measured photocurrent versus the number of photons absorbed; therefore, the ideal value of IQE is one assuming a unity quantum yield what would mean absence of internal losses. The IQE of a PQED is predictable because it can be determined only from fundamental constants (and the radiation wavelength) and the deviation from the ideal behaviour due to internal losses can be calculated.

Up to now, the IQE of a PQED radiometer is only predicted from the design and setup parameters of the two photodiodes forming this radiometer, by means of simulation software for semiconductor devices [6]. This work proposes an alternative analytical method, based on the model of the photocurrent analysis developed by Ferrero et al. [7], which considers the different internal regions of the photodiode and the characteristics of the incident beam. This work also presents the adaptation of the original model, which considers a physical junction of two semiconductors with different doping, to the self-induced junction of a PQED photodiode, caused by the surface charge resultant of growing a silicon oxide layer on the p-type doping substrate. 


\section{PQED internal quantum efficiency equation}

\section{2.a. The original model}

In order to predict the IQE of a PQED in trap configuration, it is necessary to quantify the losses due to the radiation not absorbed in the depletion region, where recombination losses are considered to be negligible. If the whole radiation was absorbed within the depletion region, for each absorbed photon a carrier would contribute to the photocurrent and the internal quantum efficiency would reach its ideal value. However, the radiation is absorbed differently within every region of the photodiode depending on its wavelength, producing differentiated surface and bulk losses.

These losses are represented in the equation of internal quantum efficiency developed by Ferrero et al.:

$$
\eta_{i}=1-\delta_{\text {out }}-\frac{\delta_{\text {bulk }}}{2}\left[1+\varepsilon_{\text {bulk }}(\lambda, T, P, S)\right]-\frac{\delta_{\text {surf }}}{2}\left[1+\varepsilon_{\text {surf }}(\lambda, T, P, S)\right] .
$$

In the following subsections the meaning of each item in equation (1) is described and how to adapt them to the self-induced photodiodes used as detectors in PQEDs is explained.

\section{2.b. Internal regions of a self-induced photodiode}

In equation (1), $\delta_{\text {surf, }}, \delta_{\text {bulk }}$ and $\delta_{\text {out }}$ represent the fractions of optical radiation not absorbed within the depletion region, i.e., the radiation absorbed at the silicon's surface, the radiation absorbed within the region of the substrate beyond the depletion region and the radiation which goes through the photodiode without being absorbed, respectively:

$$
\begin{gathered}
\delta_{\text {bulk }}=\exp \left(-\alpha_{\text {si }} x_{d, \max }\right)-\exp \left(-\alpha_{s i} H\right), \\
\delta_{\text {surf }}=1-\exp \left(-\alpha_{\text {surf }} f\right), \\
\delta_{\text {out }}=\exp \left(-\alpha_{\text {si }} H\right),
\end{gathered}
$$

where $\alpha_{s i}$ is the absorption coefficient of silicon, $x_{d, \max }$ defines the width of the depletion region, $f$ is a 'virtual thickness' of the oxide-silicon interface and $H$ is the thickness of the substrate of a self-induced photodiode, as it can be seen in Figure 1:

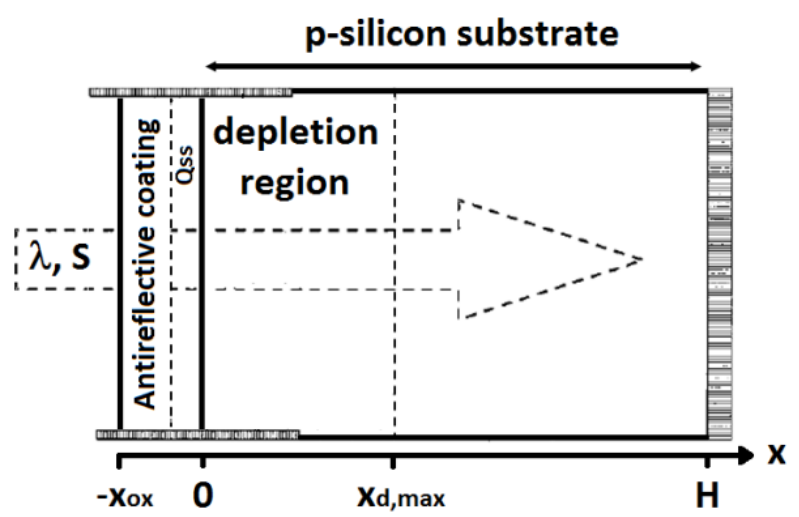

Fig. 1. Simplified diagram of a self-induced junction structure in a p-type silicon substrate. The silicon oxide layer, grown on the substrate, induces the surface charge QsS and hence, the self-induced junction and the different internal regions of the photodiode.

Since in a self-induced junction the depletion region is immediately after the oxide layer, $f$ is not a welldefined physical region and it is not represented in Fig. 1. Nevertheless, for our model it is needed a layer of certain thickness with an absorption coefficient, $\alpha_{\text {surf }}$, mimicking the oxide-silicon interface to quantify the surface losses.

\section{2.c. Probability of recombination}

The possibility of finding free recombination centres out of the depletion region determines the recombination losses. The occupancy rate depends on fractions of optical radiation not absorbed within the depletion region, temperature (through the spectral absorption coefficients) and supra-linearity factors. 
In equation (1), $\varepsilon_{b u l k}$ and $\varepsilon_{\text {surf }}$ take values between 0 and 1 , representing the probability of finding free recombination centres:

$$
\begin{gathered}
\varepsilon_{\text {bulk }}=\frac{1}{i_{\varphi} \Xi_{\text {bulk }}} \sum_{i=1}^{2} \ln \left(1+\frac{i_{\varphi} \Xi_{\text {bulk }} A_{i}}{1+\frac{i_{\varphi} \Xi_{\text {bulk }} A_{i} \delta_{\text {out }}}{\delta_{\text {bulk }}}}\right), \\
\varepsilon_{\text {surf }}=\frac{1}{i_{\varphi} \Xi_{\text {surf }}} \sum_{i=1}^{2} \ln \left(1+\frac{i_{\varphi} \Xi_{\text {surf }} A_{i}}{1+\frac{i_{\varphi} \Xi_{\text {surf }} A_{i}\left(1-\delta_{\text {surf }}\right)}{\delta_{\text {surf }}}}\right),
\end{gathered}
$$

where $i_{\phi}$ is the photocurrent that would be generated if all the radiation was absorbed within the depletion region, i.e., if for each absorbed photon a carrier contributed to the photocurrent. Hence, from the responsivity definition (the ratio between electrical response of a photodiode and the incident optical power) and with IQE equals one:

$$
i_{\varphi}=\frac{q n_{\text {air }}}{h c}(1-\rho) P \lambda
$$

where $h$ is Planck's constant, $c$ the speed of light in vacuum, $q$ the elementary charge constant, $n_{\text {air }}$ the index of refraction of air, $P$ the optical power, $\lambda$ the wavelength of the incident radiation in air and $\rho$ the reflectance.

For the sake of simplicity, equations (5) and (6) assume two identical photodiodes. $A_{i}$ is the fraction of radiation absorbed by each photodiode respect to the radiation absorbed by the PQED trap detector:

$$
A_{i}=\frac{P_{a i}}{P_{a_{P Q E D}}} .
$$

The configuration for a PQED wedged trap detector manufactured with two photodiodes is [8]:

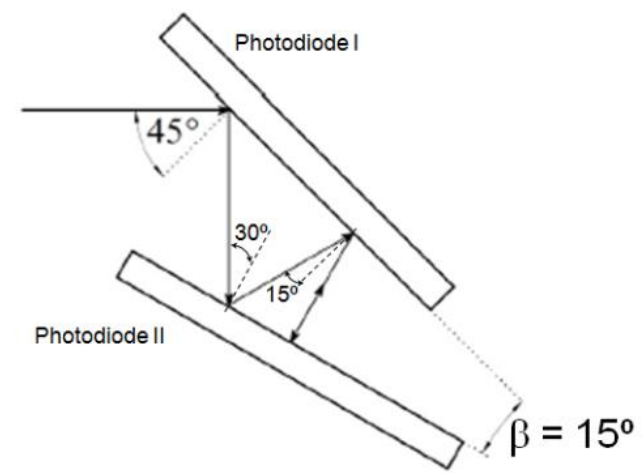

Fig. 2. Wedged trap configuration of a PQED detector with seven reflections between the photodiodes.

If the first incidence angle is $45^{\circ}$, there will be seven consecutive reflections absorbed inside the trap until returning out again, as it is represented in Fig. 2. Therefore, the optical power absorbed by photodiode I, photodiode II and PQED trap detector, respectively, can be obtained as:

$$
\begin{gathered}
P_{a_{1}}=P\left[\left(1-\rho_{45^{\circ}}\right)\left(1+\rho_{45^{\circ}} \rho_{30^{\circ}}{ }^{2} \rho_{15^{\circ}}^{2} \rho_{0^{\circ}}\right)+\left(1-\rho_{15^{\circ}}\right) \rho_{45^{\circ}} \rho_{30^{\circ}}\left(1+\rho_{15^{\circ}} \rho_{0^{\circ}}\right)\right], \\
P_{a_{2}}=P\left[\left(1-\rho_{30^{\circ}}\right) \rho_{45^{\circ}}\left(1+\rho_{30^{\circ}} \rho_{15^{\circ}}{ }^{2} \rho_{0^{\circ}}\right)+\left(1-\rho_{0^{\circ}}\right) \rho_{45^{\circ}} \rho_{30^{\circ}} \rho_{15^{\circ}}\right] \\
P_{a_{P Q E D}}=P\left[1-\rho_{45^{\circ}}{ }^{2} \rho_{30^{\circ}}{ }^{2} \rho_{15^{\circ}}{ }^{2} \rho_{0^{\circ}}\right]
\end{gathered}
$$

where $\rho_{45^{\circ}}, \rho_{30^{\circ}}, \rho_{15^{\circ}}$ and $\rho_{0}$ 。 represent the reflectance calculated by means of Fresnel's coefficients, considering the interfaces air-oxide and oxide-silicon inside the photodiodes, in every reflection inside the PQED trap detector. 


\section{2.d. Supra-linearity factors}

The internal design values of the photodiodes (charge carriers lifetime, substrate doping concentration and surface recombination velocity) and the irradiance (incident power per surface unit) are the last parameters that determine the probability of finding free recombination centres.

In equations (5) and (6), $\Xi_{\text {bulk }}$ and $\Xi_{\text {surf }}$ can be considered the bulk and surface supra-linearity factors, because they gather the material and radiation parameters and explain the possible nonlinear response of the photodiode, e. g., when the recombination centres are filled at certain irradiance levels and supraresponsivity of the photodiode appears.

Therefore, the bulk and surface supra-linearity factors can be calculated, respectively, by the following expressions:

$$
\begin{aligned}
& \Xi_{\text {bulk }}=\frac{2 \tau \alpha_{s i}}{q N_{A}} \frac{\delta_{\text {bulk }}}{S}, \\
& \Xi_{\text {surf }}=\frac{2 H x_{\text {ox }} \alpha_{\text {surf }}}{q S_{\text {eff }} Q_{S S}} \frac{\delta_{\text {surf }}}{S},
\end{aligned}
$$

where $N_{A}$ is the doping concentration, acceptor impurities in a p-type substrate of silicon, $\tau$ the lifetime of the minority carriers, $S_{\text {eff }}$ the surface recombination velocity, $Q_{S s}$ the trapped surface charge density at the oxide-silicon interface, $x_{o x}$ the width of the oxide layer and $S$ the cross-section of the beam.

\section{2.e. Biasing voltage}

For a PQED it is necessary to consider a reverse electrical bias applied to its photodiodes in order to extend the depletion layer. This effect can be included in equation (2), where the width of the depletion layer will be [9]:

$$
x_{d, \max }=\left(\frac{2 \varepsilon_{r_{s i}} \varepsilon_{0}}{q N_{A}} V_{s(i n v)}\right)^{1 / 2}
$$

with the relative permittivity of silicon, $\varepsilon_{r s i}$, the vacuum permittivity, $\varepsilon_{0}$, and the strong inversion surface potential, $V_{s(i n v)}$ which has been considered equal to the double of the bulk Fermi potential:

$$
V_{s(i n v)}=2 \frac{k_{B} T}{q} \ln \frac{N_{A}}{n_{i}},
$$

with Botlzmann's constant, $k_{B}$, intrinsic concentration of carriers, $n_{i}$ and temperature, $T$.

When a bias voltage is applied through the photodiode, the width of the depletion region changes according to the expression:

$$
\begin{gathered}
x_{d, \max (\text { bias })}=\left(\frac{2 \varepsilon_{r_{i}} \varepsilon_{0}}{q N_{A}}\left(V_{s(i n v)}-V_{\text {bias }}\right)\right)^{1 / 2}, \\
V_{\text {bias }}=-\left|V_{R}\right|,
\end{gathered}
$$

where $V_{\text {bias }}$ has negative sign for reverse bias (positive voltage applied to the $\mathrm{N}$-region of the photodiode with respect to the P-region) and positive for forward bias. Hence, a reverse bias voltage, $V_{R}$, is applied through the photodiodes of a PQED in order to increase the internal quantum efficiency by extending the depletion region.

\section{Results}

\section{3.a. Input data}

The aim of this work is to propose the analytical model as an alternative to determine the IQE of PQEDs; hence, it is required to introduce numerical values to study the behaviour of the model.

In order to apply the model described in the previous section, different parameters have to be collected, whose values, from different sources, are listed in Table 1. The refractive index and absorption coefficient of silicon and silicon oxide refractive index are calculated according to Green [10] and Herzinger [11] models; substrate thickness, minority carriers lifetime, surface recombination velocity, doping 
concentration and surface charge density are design values of the photodiodes; reverse bias voltage, optical power, beam cross section and temperature are determined from the measurement conditions and, finally, oxide-silicon interface width and absorption coefficient are parameters introduced by the analytical model and their values were estimated.

TABLE 1. Data values of internal parameters of the photodiode and incoming beam.

\begin{tabular}{|c|c|c|}
\hline \hline Parameter & Symbol & Value \\
\hline \hline Si absorption coefficient & $\alpha_{\mathrm{si}}$ & Green model \\
$\mathrm{SiO}_{2}$-Si interface absorption coefficient & $\alpha_{\mathrm{surf}}$ & $10^{-5} \alpha_{\mathrm{si}}$ \\
$\mathrm{SiO}_{2}$ refractive index & $\mathrm{n}_{\mathrm{ox}}$ & Herzinger model \\
Si refractive index & $\mathrm{n}_{\mathrm{si}}$ & Green model \\
Substrate thickness & $\mathrm{H}$ & $525 \mu \mathrm{m}$ \\
$\mathrm{SiO}_{2}$-Si interface width & $\mathrm{f}$ & $0.5 \mu \mathrm{m}$ \\
Charge carriers lifetime & $\tau$ & $0.05 \mathrm{~ms}$ and $0.4 \mathrm{~ms}$ \\
Surface recombination velocity & $\mathrm{S}_{\mathrm{eff}}$ & $3 \times 10^{4} \mathrm{~cm}_{\mathrm{s}-3 \times 10^{7} \mathrm{~cm} / \mathrm{s}}$ \\
Substrate doping concentration & $\mathrm{N}_{\mathrm{A}}$ & $2 \times 10^{12} \mathrm{~cm}^{-3} \mathrm{and} 1 \times 10^{13} \mathrm{~cm}^{-3}$ \\
Carriers intrinsic concentration & $\mathrm{n}_{\mathrm{i}}$ & $1 \times 10^{10} \mathrm{~cm}^{-3}$ \\
Surface charge density & $\mathrm{Q}_{\mathrm{ss}}$ & $6.2 \times 10^{11} \mathrm{~cm}^{-2}$ \\
Reverse bias voltage & $\mathrm{V}_{\mathrm{R}}$ & $-5 \mathrm{~V}$ and $-20 \mathrm{~V}$ \\
Optical power & $\mathrm{P}$ & $100 \mu \mathrm{W}$ and $1 \mathrm{~mW}^{-25}$ \\
Cross section of the beam & $\mathrm{S}$ & $10 \mathrm{~mm}^{2}$ \\
Temperature & $\mathrm{T}$ & $300 \mathrm{~K}$ \\
\hline \hline
\end{tabular}

\section{3.b. Spectral behaviour}

One way to illustrate the behaviour of the model is to calculate the spectral IQE. However, since a PQED is a high efficient photodetector, it is usual to represent, the complementary part of the IQE, i.e., the internal quantum deficiency (IQD=1-IQE). The following graphs represent the IQD, i.e., the internal losses, in parts per million (ppm).

The IQD results, shown in Fig. 3, have been calculated choosing a combination of parameters values, given in Table 1, to represent the corresponding minimum and maximum scenarios of losses.

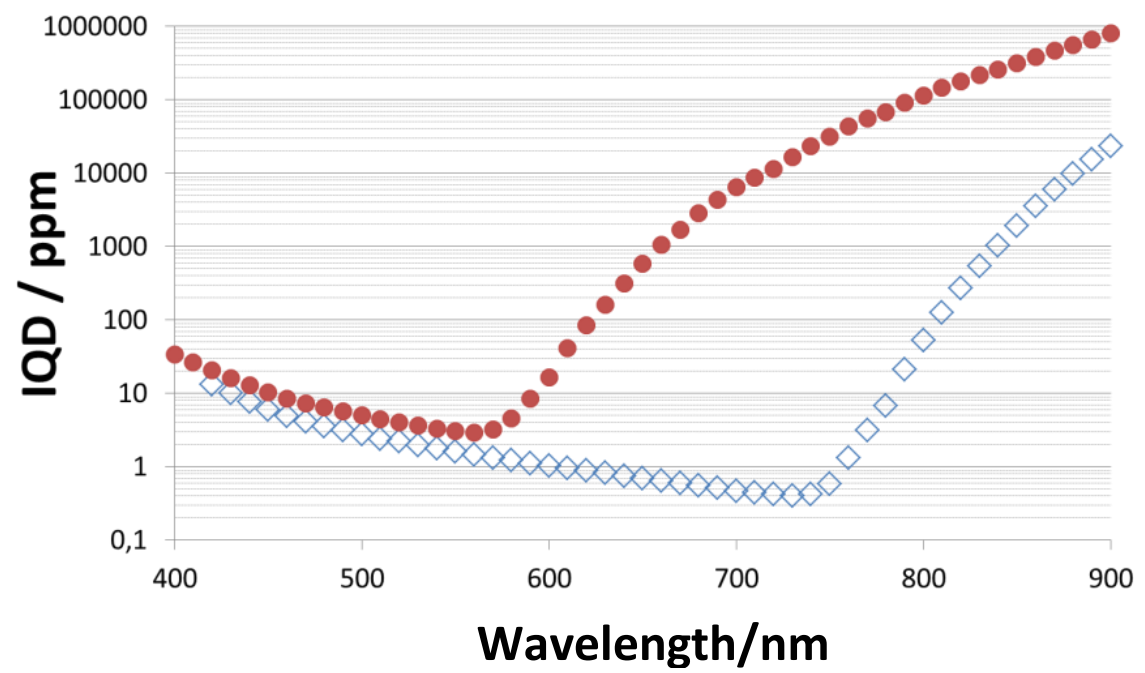

Fig. 3. IQD calculated by analytical model, at $300 \mathrm{~K}$, as a function of wavelength for PQED trap detector of two identical photodiodes with two combinations of parameters values: $1 \times 10^{13} \mathrm{~cm}^{-3}$ doping concentration of the substrate, $0.05 \mathrm{~ms}$ bulk lifetime, $3 \times 10^{7} \mathrm{~cm} / \mathrm{s}$ surface recombination velocity and reverse bias voltage of $5 \mathrm{~V}$ (filled dots); and $2 \times 10^{12} \mathrm{~cm}^{-3}$ doping concentration of the substrate, $0.4 \mathrm{~ms}$ lifetime of the charge carriers in the bulk, $3 \times 10^{4} \mathrm{~cm} / \mathrm{s}$ surface recombination velocity and reverse bias voltage of $20 \mathrm{~V}$ (empty squares).

Fig. 3 shows how the surface losses are higher at shorter wavelengths, then, as the radiation penetrates into the depletion region, the losses drop and, finally, the IQD increases again at longer wavelengths due to the bulk losses. 
Also, in the curves of the IQD in Fig. 3, three principal effects can be observed due to the changes of the reverse bias voltage, bulk doping concentration, lifetime of the charge carriers and the surface recombination velocity.

The more noticeable effect is how the IQD shifts its minimum towards longer wavelengths when the reverse bias voltage is increased and the bulk doping concentration is lower. The reason is that the IQD shifts its minimum towards longer wavelengths for a larger depletion region width, which is proportional to the square root of the reverse bias and inversely proportional to the square root of the doping concentration.

Furthermore, Fig. 3 reveals a higher slope in the curve at longer wavelengths for higher doping concentration and lower lifetime of the charge carriers. This means that the IQD is inversely proportional to the lifetime of the charge carriers and proportional to the doping concentration, since doping impurities behave as recombination centres producing bulk losses.

Moreover, at shorter wavelengths, a slight vertical shift between the two curves in Fig. 3 can be observed because the IQD increases when the surface recombination velocity is higher.

\section{3.c. Comparison with simulations}

To test the results, the IQD for a PQED trap detector calculated by the analytical model was compared with the results obtained by simulation software for semiconductor devices. Simulations for the same values of parameters, except the surface recombination velocity, were performed by JV by means of PC1D (onedimensional) and Genius (three-dimensional) software:

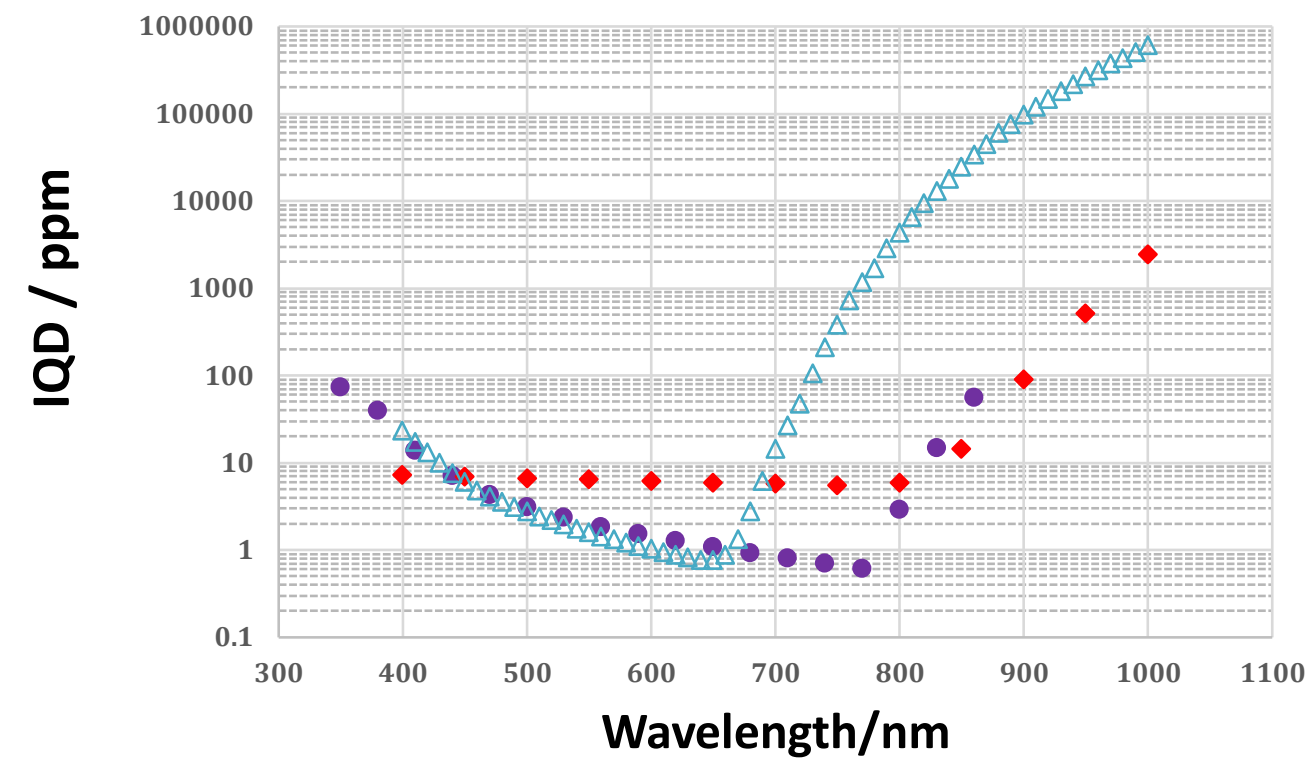

Fig. 4. Comparison of IQD calculated by the analytical model with $1000 \mathrm{~cm} / \mathrm{s}$ of surface recombination velocity (empty triangles) and software simulations performed by JV by means of PC1D with $10 \mathrm{~cm} / \mathrm{s}$ of surface recombination velocity (filled squares) and Genius with $1000 \mathrm{~cm} / \mathrm{s}$ of surface recombination velocity (filled dots), at $300 \mathrm{~K}$, as a function of wavelength for PQED trap detector of two identical photodiodes with $2 \times 10^{12} \mathrm{~cm}-3$ doping concentration of the substrate, $0.4 \mathrm{~ms}$ bulk lifetime, $5 \mathrm{~V}$ reverse bias voltage and with $100 \mu \mathrm{W}$ optical power.

Fig. 4 shows similar curves of the IQD predicted by analytical model and software simulations. The main difference observed is how the bulk losses begin to increase at shorter wavelengths for the analytical model when the radiation penetrates deeper beyond depletion region. It could be a sign of a different width of the depletion region calculated by analytical model and simulations. For future improvements, it is important to study the absorption and the width of the different layers to characterize accurately the structure of a self-induced junction of the photodiodes of a PQED and the mechanisms of generation of carriers and the losses within each region. Nevertheless, the IQD results predicted by the analytical model and simulations are in the same range of losses for wavelengths between $400 \mathrm{~nm}$ and $700 \mathrm{~nm}$. 


\section{3.d. Irradiance dependence}

The last feature of the analytical model can inform about how the IQD depends on the irradiance. A decrease of losses with higher irradiance is observed at certain levels of optical power in photodiodes due to the supra-responsivity.

Fig. 5 shows how the IQD decreases slightly with a higher value of power, $1 \mathrm{~mW}$ with a constant value of the cross section of the beam, because recombination centres start to be filled at this level of power and some of the excited carriers beyond the depletion region reach it causing the supra-responsivity of the detector.

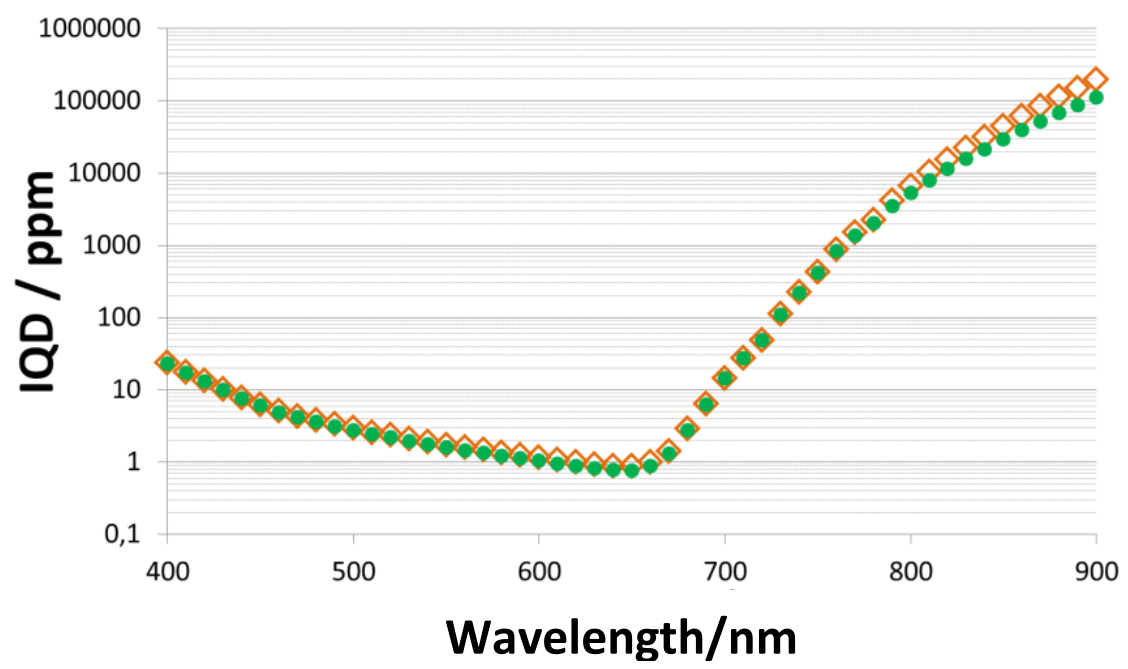

Fig. 5. IQD calculated by CSIC model, at $300 \mathrm{~K}$, as a function of wavelength for PQED trap detector of two identical photodiodes with $2 \times 10^{12} \mathrm{~cm}-3$ doping concentration of the substrate, $0.4 \mathrm{~ms}$ bulk lifetime, $3 \times 10^{5} \mathrm{~cm} / \mathrm{s}$ surface recombination velocity, $5 \mathrm{~V}$ reverse bias voltage and with $100 \mu \mathrm{W}$ optical power (empty squares); and the same conditions with $1 \mathrm{~mW}$ optical power (filled dots).

\section{Conclusions}

An analytical model to predict the IQE of a PQED radiometer has been developed by adapting the Ferrero et al. model to the self-induced junction of a PQED photodiode. The analytical model determines, quantitatively, how the design and manufacturing parameters of the photodiodes contribute to the IQE. This work proposes the analytical model as an alternative method to predict the IQE of a PQED radiometer, since its behaviour is similar to the software simulations. The IQE grows with the lifetime of the charge carriers and the reverse bias voltage applied to the photodiodes, while IQE decreases when the doping concentration and the surface recombination velocity are increased.

The main difference between the behaviour of the analytical model and the simulations is the growing of the bulk losses at long wavelengths predicted by the analytical model. Nevertheless, the IQD results predicted by the analytical model and simulations are in the same range of losses for wavelengths between $400 \mathrm{~nm}$ and $700 \mathrm{~nm}$.

Furthermore, as a consequence of the IQE dependence on the power and the cross section of the incoming beam, the model predicts an increase of the IQE with the irradiance, at certain levels of optical power, due to the supra-responsivity of the photodiode when recombination centres begin to be filled. This feature could be useful to determine the linear range of response of a PQED.

For future improvements, it is required to study the absorption and the depth of the different layers in order to recreate accurately the self-induced junction structure of the photodiodes of a PQED and the mechanisms of generation of carriers and the losses within each region. 


\section{Acknowledgements}

This work has been supported by the European Commission through the project SIB57-NEWSTAR under the European Metrology Research Programme (EMRP). The EMRP is jointly funded by the EMRP participating countries within EURAMET and the European Union. The authors of CSIC acknowledge support from the Comunidad de Madrid and the European Union under program SINFOTON-CM (S2013/MIT-2790).

Parts of this work have been presented at IX Reunión Iberoamericana de Óptica in Modelo analítico para la determinación de la eficiencia cuántica interna de un detector de eficiencia cuántica interna (PQED), 2016. 